\title{
Extractive text Summarization using Genetic Clustering Algorithm
}

\author{
Alok Rai ${ }^{1}$, Yashashree Patil ${ }^{2}$, Pooja Sulakhe ${ }^{3}$, Gaurav Lal ${ }^{4}$ \\ Student, IT Dept, NBNSSOE, Pune, India ${ }^{1,2,3,4}$
}

\begin{abstract}
Text summarisation is largely summarizing the source text into a simplified short version maintaining its actual information content and also the abstract that means. Attributable to apace growing use of internet, the globe is additionally facing drawback to tackle with dangerous quantity of data often facet by facet in kind of text .The bountiful offer of data generally cause time delay within the search of information recovery. In this relative concern automatic text summarisation has a crucial issue concerning data recovery of time. Manually summarizing giant document of text is incredibly troublesome task for individual. For this, extractive summarizing tool supported verified algorithm is required. Therefore supported the analysis of already planned model of extractive text summarisation, We are developing extractive text summarization tool based on genetic algorithm named AETS.. This approach are valid victimization normal information sets and quality measures.
\end{abstract}

Keywords: Feature extraction, Text summarization, Part of speech, Automatic text, Semantic nets.

\section{INTRODUCTION}

In this internet era, lot of rough-textured information is 2] Rajesh S.Prasad, U. V. Kulkarni, and Jayashree. R. obtainable on network for user. Volume of this Prasad "Connectionist Approach to Generic Text information is just too huge to store and handle. User Summarization" scholar.waset.org/1999.4/1999, 2009

won't be ready to summarize this information in - An Evolving connectionist System that is adaptive, economical manner. Human being can summarize simply incremental learning and knowledge representation system information with their own thoughts and with their that evolves its structure and functionality

additional content. Text extraction of machine isn't thus effective like human thinking.

3] Rajesh Shardanand Prasad and Uday Kulkarni Summarization done by machine is theoretical and report Implementation and Evaluation of Evolutionary by human is an extractive outline. Text summarization Connectionist Approaches to Automated Text ought to be temporary enough however embody whole Summarization. 1366-1376, 2010.

which means of original information. It plays very -A new text summarizer based on fuzzy logic. important role to extract helpful information. It's helpful in convalescent data for user.

Hence, we tend to propose a technique to expeditiously summarized text so as to induce sorted information. In this paper, we tend to contemplate the system of text Summarization as Evolving system that learns incrementally through expertise within the surroundings.

Text Summarization will be outlined as "extracting brief 5] Rajesh S.Prasad, Dr.U.V.Kulkarni "A Novel

and correct information from given information, which is Evolutionary Connectionist Text Summarizer" 2008.

able to be satisfy to user". Open text Summarization tool is employed by windows and UNIX.

\section{LITERATURE SURVEY}

From last few years, problem of text summarization increases. In order to tackle with this problem various techniques are proposed.

1] René Arnulfo García-Hernández and YuliaLedeneva "Single Extractive Text SummarizationBased on a Genetic Algorithm” MCPR 2013, LNCS 7914, pp. 374-383, 2013. -A genetic algorithm is proposed with special emphasis on the fitness function which permits to contribute with some conclusions.

4] Ladda Suanmali, Naomie Salim, Mohammed Salem Binwahlan "Automatic text summarization using featured based fuzzy extraction" Bil 2, December 2008.

- Automatic text summerization by sentence with important features based on fuzzy logic

- An Evolving connectionist System that is adaptive, incremental learning and knowledge representation system that evolves its structure and functionality.

\section{RELATED WORK}

The extractive summaries are the ones which can be composed through precise phrases or phrases which are gift within the supply textual content. Then the problem of achieve extractive summariesfrom the bottom-text is reduced to discover the smallest set of sentences that constitute the entire text appropriately.

In practice, the extractive summaries are limited by means of size; for example, an extractive precise need to now not 
Vol. 5, Issue 12, December 2016

be longer than the $10 \%$ of the entire textual content where the period of the precise is calculated by means of the number of words. This implies that for actual troubles extractive summaries are definitely the first-rate feasible approximation of the base-textual content which fulfils the defined précis-constraints.

\section{FEATURES}

For calculating summery, it is essential to represent sentences within the vector shape with the intention to offer us and attributes so one can constitute the input records. For our need, we are going to use 5 features to extract the exact sentences from input data and so that it will provide output as ' 0 'or ' 1 '.

1)Text Summarization:It can be calculated by using the ratio of number of titles in words by number of word in title.

$$
\text { output }=\frac{\text { Number of titles in word }}{\text { Number of word in tilte }}
$$

2) Sentence length : It can be calculated in order to find out short sentences such as book name, authors, sub meaning s .It can be calculated as,

$$
\text { output }=\frac{\text { number of words in sentence }}{\text { number of words in longest sentence }}
$$

3) Term weight:Weight age of sentence means importance's of sentences in document. It can be calculated by taking occurrences of sentence in document. It can be calculated by,

$$
\text { output }=\frac{\text { Sum of TF ISF }}{\text { Max(sum of TS ISF }}
$$

Where TS-ISF is nothing but term Frequency and ISF is Inverse term frequency.

4) Sentence position: Position of sentence is an important for deciding the importance of sentence. So for the first sentence output will be 1 and for other it will be 0 .

5)Sentence to sentence similarity: There may be a different sentence with same meaning,Sowe can extract the content by neglecting such sentences. It can be calculated like

$$
\text { output }=\frac{\text { Sentence similarity in document }}{\text { Max(sum of sentence similarity) }}
$$

\section{Properties}

In this paper, five features are utilized in order to score each sentence. These features are:-

- Words similarity

- Repetitive sentence

- Term weight

- Title features

\section{GENETIC ALGORITHM}

Genetic algorithms are stochastic search strategies coping with a population of simultaneous seek positions. A traditional genetic algorithm consists of three important elements:

- A coding of the optimization problem

- A mutation operator

-A hard and fast of facts-exchange operators

The coding of the optimization hassle produces the required discretization of the variable values (for optimization of actual functions) and makes their easy management in a population of search factors viable. Generally the maximum variety of seek points, i.e., the population size, is constant at the start. The mutation operator determines the opportunity with which the facts structures are changed. This may arise spontaneously (as in stochastic search) or handiest whilst the strings are combined to generate a brand new populace of seek points. In binary strings a mutation corresponds to a bit flip. The records trade operators manipulate the recombination of the hunt points which will generate a brand new, better population of factors at each new release step. Earlier than recombining, the feature to be optimized must be evaluated for all records structures in the population. The search points are then looked after inside the order of their function fee, i.e., within the order of their so-called fitness. Ina minimization problem the points which might be located at the beginning of the listing are the ones for which the characteristic fee is lowest. The ones points for which the feature to be minimized has the best characteristic cost are placed on the give up of the listing. Genetic algorithm is the search method in which principle of natural selection and genetics is used. Solutions for the problem is considered as set of candidate solutions. This candidate solution is know as "chromosomes". And the alphabets from the string is called as genes and the values of genes are know as alleles. Take an example, whiletravelling salesman problem. In this problem chromosomes is nothing but the route. And gene are nothing but city.

\section{- Initialization}

The initial population of candidate solutions is usually generated randomly across the search space. However, domain-specific knowledge or other information can be easily incorporated.

\section{- Evolution}

Once the population is initialized or an offspring population is created, the fitness values of the candidate solutions are evaluated. Selection allocates more copies of those solutions with higher

\section{- Selection}

Selection allocates more copies of those solutions with higherfitness values and thus imposes the survival-of-the- 
fittest mechanism onthe candidate solutions. The main idea of selection is to prefer better solutions to worse ones, and many selection procedures have beenproposed to accomplish this idea, including roulette-wheel selection,stochastic universal selection, ranking selection and tournament selection

\section{- Recombination}

Recombination combines parts of two or more parental solutions to create new, possibly better solutions (i.e. offspring). There are many ways of accomplishing this (some of which are discussed in the next section), and competent performance depends on a properly designed recombination mechanism. The offspring under recombination will not be identical to any particular parent and will instead combine parental traits in a novel manner

\section{- Mutation}

While recombination operates on two or more parental chromosomes, mutation locally but randomly modifies a solution. Again, there are many variations of mutation, but it usually involves one or more

\section{Properties of genetic algorithm:-}

Genetic algorithms have made a real impact on all those problems in which there is not enough information to build a differentiable function or where the problem has such a complex structure that the interplay of different parameters in the final cost function cannot be expressed analytically. Following are the properties of genetic algorithm.

- Selection of parents string

Selection with replacement is used, i.e., the whole population is the basis for each individual parent selection. It can occur that the same string is selected twice. The probability $\mathrm{P}$ that a string is selected which contains the bit pattern

\section{- Recombination}

For the recombination of two strings a cut-off point is selected between the two positions and then a crossover is carried out. The probability that a schema is transmitted to the new string depends on two cases. If both parent strings contain, then they pass on this substring to the new string. If only one of the strings contains, then the schema is inherited at most half of the time.

\section{- Mutation}

When two strings are recombined, the information contained in them is copied bit by bit to the child string. A mutation can produce a bit flip with the probability. This means that a schema with fixed bits will be preserved after copying with probability.

In genetic algorithm, its mainly focuses on the population of candidate solution. This is user defined parameter which will effects on factors such as scalability and performance of algorithm. We can solve the problem with the following steps

\section{DIAGRAM}

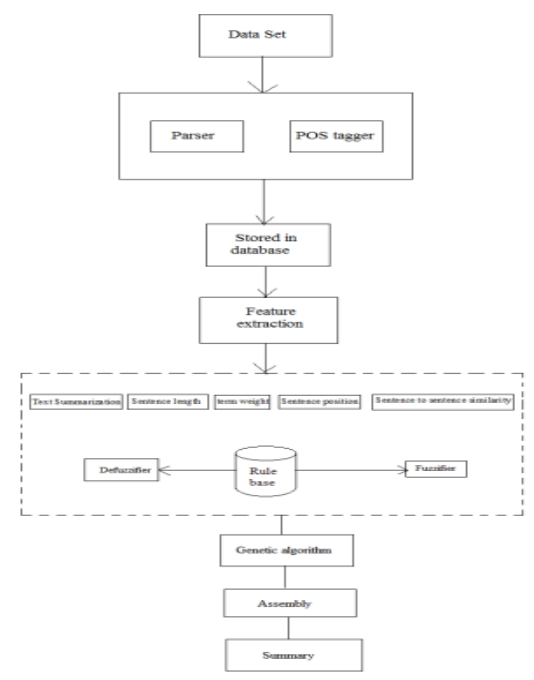

Fig:- Architectural diagram

\section{CONCLUSION}

As visible from the results genetic algorithm is sentence choice-based technique. Text summarization is the method of reducing a text file with the help of genetic algorithm retains the most essential textual content of the any authentic file. Summarization is coming of age of filtering the word. For preferred domain names is sentence extraction and highlight most effective importance text which we need. The use of genetic algorithm we can put into effect language modelling, multilingual summaries, summarization of email, spoken document summarization also. Using text summarization, spotting portions of the summary that in shape the input files. We will exact relate to the examiner an existing summarizer. Genetic algorithm helps to build a summarizer from scratch and separated it in to highlight form. Broaden a summarization assessment toolkit permitting comparisons between extractive and non-extractive summaries produce an annotated corpus for further studies in text summarization. This method utilized in documentation then it's far very time saving of human users. The summarization approach calculates the frequencies of the key word within the sentence it constitute where those sentence are gift then tag that text

\section{REFERENCES}

[1] René Arnulfo García-Hernández and Yulia Ledeneva "Single Extractive Text SummarizationBased on a Genetic Algorithm" MCPR 2013, LNCS 7914, pp. 374-383, 2013.

[2] Rajesh S.Prasad, U. V. Kulkarni, and Jayashree.R.Prasad "Connectionist Approach to Generic Text Summarization" scholar.waset.org/1999.4/1999, 2009

[3] Rajesh Shardanand Prasad and Uday Kulkarni Implementation and Evaluation of Evolutionary Connectionist Approaches to Automated Text Summarization. 1366-1376, 2010

[4] Ladda Suanmali, Naomie Salim, Mohammed Salem Binwahlan "Automatic text summarization using featured based fuzzy extraction" Bil 2 , December 2008

[5] Rajesh S.Prasad, Dr.U.V.Kulkarni "A Novel Evolutionary Connectionist TextSummarizer" 2008 